\title{
Image and video quality improvement techniques for emerging applications
}

\author{
Volodymyr Ponomaryov ${ }^{1 *}$, Thorsten Herfet ${ }^{2}$, Vladimir Lukin ${ }^{3}$, Bogdan Smolka ${ }^{4}$ and Vladimir Zlokolica ${ }^{5}$
}

Nowadays, new "digital" areas have brought in many new image and video applications and new technologies in different fields, such as biology, medicine, engineering, and entertainment. The images and videos are compressed, transmitted, captured, and stored in various digital forms, with different types and amounts of impaired artifacts. While in bio-medical applications, speckle noise and coding artifacts are most common, in the entertainment and engineering applications, the digital coding and transmission artifacts are considered as dominant. Furthermore, each of the emerging applications and technologies has introduced different kinds of specific, correlated/structured distortions. The removal of such distortions, i.e., denoising, has not sufficiently been investigated and needs to be further explored. This is especially true having in mind consistent growth of new emerging digital multimedia applications and services.

This Special Issue is dedicated to novel image and video quality assessment and improvement techniques and systems which are aimed for currently challenging applications in which the visual quality is critical. Thirteen outstanding research articles, regarding this topic, are published in this issue and cover four important topics:

- Novel image and video processing optimization algorithms based on objective and subjective image quality assessment.

- Image/video enhancement and analysis for 3D visualization.

- Image and video enhancement and analysis for artifact reduction.

- Image and video analysis for emerging applications.

The first topic is covered in the following four research articles.

In the first contributed article entitled "Efficiency analysis of color image filtering", by D. Fevralev, N.

\footnotetext{
* Correspondence: vponomar@ipn.mx

${ }^{1}$ National Polytechnic Institute, Santa Ana 1000, Mexico-city, 04430, Mexico
} Full list of author information is available at the end of the article
Ponomarenko, V. Lukin, S. Abramov, K. Egiazarian, and J. Astola, the authors address the conditions under which filtering can visibly improve the image quality. Using color image database TID2008, the filter efficiency for images corrupted by different noise types is analyzed. The limit of filtering efficiency is determined for independent and identically distributed (i.i.d.) additive noise and compared to the output mean square error of stateof-the-art filters. Among the component-wise and vector de-noising techniques studied, the latter approach is demonstrated to be more efficient. Using modern visual quality metrics, it has been determined, for which levels of i.i.d. and spatially correlated noise, the noise in original images or residual noise and distortions in filtering images are practically invisible.

In the second article entitled "No-reference image quality metric based on image classification", by $\mathrm{H}$. Choi and C. Lee, the authors present a new no-reference objective image quality metrics (blocking and blur metrics) based on image classification. The blocking metric is computed by considering that the visibility of horizontal and vertical blocking artifacts can change depending on background luminance levels. The blur metric takes into account the fact that blurring in edge regions is generally more sensitive to the human visual system. Experimental results show that each metric correlates well with subjective ratings, demonstrating that the proposed image quality metrics consistently provide good performance with various types of content and distortions.

In the third article entitled "A Reduced-Reference Perceptual Image and Video Quality Metric based on Edge Preservation" by M. Martini, B. Villarini, and F. Fiorucci, the observation that the human eye is very sensitive to edge and contour information of an image underpins the proposal of novel reduced reference (RR) quality metric, which compares edge information between the distorted and the original images. The results highlight that the proposed metric correlates well with subjective observations, also in comparison with commonly used full-reference metrics and with a state-of-the-art metric. 
In the final article of this topic entitled "Automated Optical Inspection System for Digital TV Sets", by I. Kastelan, M. Katona, D. Marijan, and J. Zloh, the author introduce a real-time test and verification system for full-reference automatic image quality assessment and verification of digital TV sets. The test is executed in three steps: image acquisition by camera, TV screen content extraction, and full-reference image quality assessment. The proposed system is used to automate the verification step on the final production line of digital TV sets. The time required for verification step decreased by a factor of 5 , when using the proposed system on the final production line instead of a manual one.

In addition to image and video processing algorithm optimization using different image quality metrics, the image/video enhancement and analysis for 3D visualization plays an important role in image and video quality improvement techniques. This topic is discussed in three following articles.

The article entitled "Denoising Algorithm for the $3 D$ Depth Map Sequences Based on Multihypothesis Motion Estimation" by L. Jovanov, A. Pizurica, and W. Philips proposes an efficient wavelet-based depth video denoising approach based on multi-hypothesis motion estimation aimed specifically at time-of-flight depth cameras. The proposed algorithm performs a search for the most similar blocks from the surrounding depth video frames, of the current noisy depth frame, using both texture information from the accompanying video sequence, and the depth sequence. Then, the algorithm performs motion compensated filtering which uses motion estimation reliabilities from the motion estimation step. Finally, the algorithm performs adaptive spatial filtering of the depth map (DM) to remove the remaining noise after temporal filtering. The presented denoising results justify that the proposed method outperforms recently proposed depth sequence denoising methods.

The authors S. Smirnov, A. Gotchev, and K. Egiazarian in the article entitled "Methods for depth-map filtering in view-plus-depth $3 D$ video representation" study the problem of post-processing of DM degraded by improper estimation or by block-transform-based compression. A number of post-filtering methods are modified and compared for their applicability to the task of DM restoration and post-filtering. It has been developed an efficient implementation for filtering DM sequences demonstrating high-quality and time-consistent DMs.

Finally, the authors E. Ramos-Diaz, V. Kravchenko, and V. Ponomaryov in the article entitled "Efficient $2 D$ to $3 D$ video conversion implemented on DSP" present an efficient framework to generate $3 \mathrm{D}$ video sequences. This algorithm is based on a DM computation employing wavelet functions technique at several decomposition levels in processing a 2D video sequence, finally applying DM in anaglyph synthesis for a 3D video sequence reconstruction. The proposed approach exhibits better performance according to the commonly used metrics (structural similarity and quantity of bad disparities) in comparison with existing techniques. The hardware implementation justifies the possibility of realtime visualization for 3D color video sequences.

The third topic, image and video enhancement and analysis in suppression of artifacts is discussed in following two articles. In "A study on the impact of $A L-F E C$ techniques on TV over IP Quality of Experience", by F. Battisti, M. Carli, E. Mammi, and A. Neri, the evaluation of the effectiveness of Application Layer-Forward Error Correction (AL-FEC) scheme in video communications over unreliable channels is presented. One objective consists in verification of the effectiveness of AL-FEC techniques in terms of perceived Quality of Service (QoS). Another objective is evaluation of the trade-off between AL-FEC redundancy and video quality degradation for a given packet loss ratio. Three quality metrics are used, namely the full-reference objective quality metric NTIA-VQM combined with the ITU-T Rec. G.1070, the full-reference DMOS-KPN metric, and the pixel-wise error comparison performed by using the PSNR distortion measure. A post-processing synchronization between the original and the reconstructed streams has also been designed for improving the fidelity of the quality measures. The experimental results prove the effectiveness of the AL-FEC scheme.

In the second article of this topic "Efficient Replicated Data for the Delivery of High-quality Video Content over P2P VoD Advertising Networks" by the authors ChienPeng Ho, Suh-Yin Lee, and Jen-Yu Yu, as a response to the demands in video quality improvement technologies, the proposed framework aims to achieve a high degree of user satisfaction for perceived video quality and to effectively utilize available resources on P2P VoD services. A P2P VoD advertising framework based on video distortion estimation prior to data stream-chunk replication is proposed. The results revealed that novel strategy (a) achieves load balance by adjusting the replication level to each candidate group according to its distortion extent, and (b) can be effective in promoting advertising to the public as an efficient commercial tool.

Finally, the fourth topic connected with image and video analysis for emerging applications is presented in three following articles. In first article of this topic entitled "Improvement for detection of microcalcifications (MC) through clustering algorithms and artificial neural networks", the authors J. Quintanilla-Dominguez, B. Ojeda-Magana, A. Marcano-Cedeno, M. CortinaJanuchs, A. Vega-Corona, and D. Andina propose a new method for detecting MCs in regions of interest 
extracted from digitized mammograms. The top-hat transform technique is used to perform contrast enhancement of the MCs. In detection stage, a novel image sub-segmentation approach based on the possibilistic fuzzy c-means algorithm is used. The mean and standard deviation were employed as an input vector in an artificial neural network (NN) classifier to identify patterns belonging to MCs and healthy tissue.

The authors Y. Shkvarko, S. Santos, and J. Tuxpan in the article entitled "Resolution-enhanced radar/SAR imaging: an experiment design framework combined with neural network-adapted variational analysis regularization" introduced the convex optimization-based descriptive experiment design regularization (DEDR) method that is aggregated with the $\mathrm{NN}$-adapted variational analysis (VA) approach for high-resolution sensing using radar/SAR image formation in uncertain operational scenarios, adaptive despeckling and dynamic scene image enhancement. The DEDR-VA-NN method outperforms the existing radar imaging techniques both in resolution and convergence rate. The simulation examples are incorporated to illustrate the efficiency of the proposed imaging techniques.

Finally, the authors A. Rehman, M. Rostami, Z. Wang, D. Brunet, and E. Vrscay in the article entitled "SSIMinspired image restoration using sparse representation" use the Structural Similarity (SSIM) index by incorporating it into the framework of sparse signal representation and approximation. A gradient descent algorithm is developed to achieve SSIM-optimal compromise in combining the input and sparse dictionary reconstructed images. The performance of the proposed method is demonstrated in image denoising and super-resolution applications. The experimental results show that the proposed SSIM-based sparse representation algorithm achieves better performance and better visual quality than the corresponding least square-based method.

We hope that the readers of this selection of articles over the broad topic of Image and Video Quality Improvement will be as excited as we have been during the editorial process.

\section{Acknowledgements}

We would like to thank the authors for their interest in this special issue, their cooperation, and their outstanding contributions, which are reflected so well in this issue. As usual, this special issue would have not been possible without the support of reviewers, whose efforts help us maintain the high standards of the EURASIP Journal; we also thank them. We also thank our Editor, Prof. Phillip Regalia, and the entire Publication Staff at Hindawi and BioMed for their dedication and hard work.

\section{Competing interests}

The authors declare that they have no competing interests.

\section{Author details}

${ }^{1}$ National Polytechnic Institute, Santa Ana 1000, Mexico-city, 04430, Mexico ${ }^{2}$ Intel Visual Computing Institute, Saarland University, Campus E2 1; 1.14,
66123 Saarbrücken, Germany ${ }^{3}$ National Aerospace University (KHAl), Chkalov 17, Kharkov, 61070, Ukraine ${ }^{4}$ Silesian University of Technology, Akademicka 16, Gliwice, 44-100, Poland ${ }^{5}$ RT-RK Computer Based Systems, Fruskogorska 11, 21000, Novi Sad, Serbia

Received: 27 December 2011 Accepted: 15 February 2012

Published: 15 February 2012

doi:10.1186/1687-6180-2012-33

Cite this article as: Ponomaryov et al: Image and video quality improvement techniques for emerging applications. EURASIP Journal on Advances in Signal Processing 2012 2012:33.

\section{Submit your manuscript to a SpringerOpen ${ }^{\circ}$ journal and benefit from:}

- Convenient online submission

- Rigorous peer review

- Immediate publication on acceptance

- Open access: articles freely available online

- High visibility within the field

- Retaining the copyright to your article

Submit your next manuscript at $\gg$ springeropen.com 